Теорія Ймовір. та Матем. Статист. Вип. 77, 2007
Theor. Probability and Math. Statist.

No. 77, 2008, Pages 177-189 S 0094-9000(09)00756-X

Article electronically published on January 21, 2009

\title{
A LOCATION INVARIANT MOMENT-TYPE ESTIMATOR II
}

\author{
UDC 519.21
}

CHENG-XIU LING, ZUOXIANG PENG, AND SARALEES NADARAJAH

\begin{abstract}
The moment estimator (Dekkers et al. (1989)) has been used in extreme value theory to estimate the tail index, but it is not location invariant. The location invariant Hill-type estimator (Fraga Alves (2001)) is only suitable for estimating positive indices. In this paper, a new moment-type estimator is studied, which is location invariant. This new estimator is based on the original moment-type estimator, but it is made location invariant by a random shift. Its asymptotic normality is derived, in a semiparametric setup.
\end{abstract}

\section{INTRODUCTION}

Suppose $X_{1}, X_{2}, \ldots, X_{n}$ are i.i.d. random variables with common d.f. $F(x)$, and let $X_{1, n} \leq X_{2, n} \leq \cdots \leq X_{n, n}$ be the associated order statistics. If there exist $a_{n}>0, b_{n} \in \mathbb{R}$ and some nondegenerate distribution $G(x)$ such that

$$
\mathrm{P}\left(X_{n, n} \leq a_{n} x+b_{n}\right)=F^{n}\left(a_{n} x+b_{n}\right) \stackrel{d}{\rightarrow} G(x) \quad \text { as } n \rightarrow \infty,
$$

then $G(x)$ must be equivalent to

$$
G_{\gamma}(x)= \begin{cases}\exp \left\{-(1+\gamma x)^{-1 / \gamma}\right\}, & 1+\gamma x>0, \gamma \neq 0 \\ \exp \{-\exp (-x)\}, & x \in \mathbb{R}, \gamma=0 .\end{cases}
$$

If $F$ satisfies (1.1), we say that $F(x)$ belongs to the domain of attraction of an extreme value of d.f. $G_{\gamma}$, denoted by $F \in D\left(G_{\gamma}\right)$, and $\gamma$ is referred to as the extreme value index (EVI). In the last two decades many estimators of the extreme value index $\gamma \in \mathbb{R}$ have been proposed that use upper order statistics; see, for example, Hill [11, Pickands [16], Dekkers et al. 4], and Drees [5]. For maximum likelihood estimators of $\gamma$, see Hall [10, Smith [20, 21], and Smith and Weissman 22]. For $\gamma>0$, Hill [11] proposed the estimator given by

$$
\hat{\gamma}_{n}^{H}(k)=\frac{1}{k} \sum_{i=0}^{k-1} \log X_{n-i, n}-\log X_{n-k, n}
$$

for $k=1, \ldots, n-1$. For $\gamma \in \mathbb{R}$, Dekkers et al. 4 proposed the moment-type estimators

$$
\hat{\gamma}_{n}^{M}=M_{n}^{(1)}+1-\frac{1}{2}\left\{1-\frac{\left(M_{n}^{(1)}\right)^{2}}{M_{n}^{(2)}}\right\}^{-1},
$$

2000 Mathematics Subject Classification. Primary 60F99.

Key words and phrases. Extreme value index, location invariant property, moment estimation, asymptotic normality, order statistics, regular varying functions. 
where

$$
M_{n}^{(j)}=\frac{1}{k} \sum_{i=0}^{k-1}\left(\log \frac{X_{n-i, n}}{X_{n-k, n}}\right)^{j}
$$

for $j=1,2$. Pan [12] discussed the asymptotic expansion of the distribution of the Hill and moment estimators. Cheng and Pan [1] and Cuntz et al. 2] obtained penultimate forms of the Hill estimator.

The above estimators are scale invariant but not location invariant. Indeed, there are mathematical as well as practical reasons to require location invariance properties. Since an affine transformation of the r.v.'s $X_{i}$ merely leads to a change of the normalizing constants $a_{n}$ and $b_{n}$, it influences neither the extreme value index nor the accuracy of the approximation (1.1). Moreover, in practice, the observations (e.g., sea levels, temperatures) depend on an arbitrarily chosen zero-point, which equally should not affect the estimator. In fact, the prominent Pickands [16] estimator given by

$$
\hat{\gamma}_{n}^{P}=\frac{1}{\log 2} \log \frac{X_{n-k+1, n}-X_{n-2 k+1, n}}{X_{n-2 k+1, n}-X_{n-4 k+1, n}}
$$

is both scale and location invariant, where $k=k(n)$ is an intermediate integer sequence, i.e., $k=k(n) \rightarrow \infty$ and $k / n \rightarrow 0$. Dekkers et al. [4, Qi and Cheng [17, and Peng 14 discussed the asymptotic behavior of various Pickands-type estimators. Segers 19 ] proposed a general Pickands estimator given by

$$
\hat{\gamma}_{n, k}(c, v)=\frac{1}{\log v} \log \left(\frac{X_{n-[c k], n}-X_{n-k, n}}{X_{n-[c v k], n}-X_{n-[v k], n}}\right),
$$

proved its consistency and asymptotic normality, and discussed the optimal choice of $c$ and $v$ in the sense of minimum square error (MSE). Drees [5] proposed a general class of estimators which have the scale invariant property. For $\gamma>0$, Fraga Alves [7] established a location invariant Hill estimator given by

$$
\hat{\gamma}_{n}^{H}\left(k_{0}, k\right)=\frac{1}{k_{0}} \sum_{i=0}^{k_{0}-1} \log \left(\frac{X_{n-i, n}-X_{n-k, n}}{X_{n-k_{0}, n}-X_{n-k, n}}\right),
$$

where $k \rightarrow \infty, k_{0} \rightarrow \infty, k / n \rightarrow 0, k_{0} / k \rightarrow 0$, and discussed its weak consistency, asymptotic expansion and the optimal choice of sample fraction $k_{0}$.

Although both (1.4) and (1.5) are location invariant, (1.4) has poor efficiency and it is difficult to decide on the optimal sample fraction $k$. Also (1.5) is only valid for $\gamma>0$. In this paper, we propose a general estimator for $\gamma \in \mathbb{R}$ based on the invariant Hill estimator and the moment-type estimator. It is given by

$$
\hat{\gamma}_{n}^{M}\left(k_{0}, k\right)=M_{n}^{(1)}\left(k_{0}, k\right)+1-\frac{1}{2}\left\{1-\frac{\left(M_{n}^{(1)}\left(k_{0}, k\right)\right)^{2}}{M_{n}^{(2)}\left(k_{0}, k\right)}\right\}^{-1},
$$

where

$$
M_{n}^{(j)}\left(k_{0}, k\right)=\frac{1}{k_{0}} \sum_{i=0}^{k_{0}-1}\left(\log \frac{X_{n-i, n}-X_{n-k, n}}{X_{n-k_{0}, n}-X_{n-k, n}}\right)^{j}
$$

for $j=1,2$ and $k=k(n), k_{0}=k_{0}(n)$ are integer sequences that satisfy $0<k \leq n$, $0<k_{0} \leq k$. We derive various properties of this new estimator: expansion under the second order regular variation condition (Section 2), necessary and sufficient conditions for asymptotic normality (Section 2), and distribution expansions (Section 2). 


\section{AsYmptotic NORMALiTy}

In order to obtain asymptotic normality of the proposed estimator, one must limit the convergence rate of (1.1). In general, one can obtain asymptotic normality under the following second order conditions.

Second order conditions. Suppose that there exists $b(t) \rightarrow 0$ with constant sign near infinity such that

(1) For $\gamma>0, \rho<0$ and $x>0$,

$$
\frac{1}{b(t)}\left\{\frac{U(t x)}{U(t)}-x^{\gamma}\right\} \rightarrow x^{\gamma} \frac{x^{\rho}-1}{\rho}
$$

as $t \rightarrow \infty$.

(2) For $\gamma<0$ (note that $U(\infty)<\infty), \rho<0$, and $x>0$,

$$
\frac{1}{b(t)}\left\{\frac{U(\infty)-U(t x)}{U(\infty)-U(t)}-x^{\gamma}\right\} \rightarrow x^{\gamma} \frac{x^{\rho}-1}{\rho}
$$

as $t \rightarrow \infty$.

(3) For $\gamma=0, \rho<0$ and $x>0$,

$$
\frac{1}{b(t)}\left\{\frac{U(t x)-U(t)}{a(t)}-\log x\right\} \rightarrow \frac{x^{\rho}-1}{\rho}
$$

with

as $t \rightarrow \infty$.

$$
\frac{1}{b(t)}\left\{\frac{a(t x)}{a(t)}-1\right\} \rightarrow 0
$$

For these three cases, one can easily infer that $|b(t)| \in R V_{\rho}$. We call $b(t)$ the second auxiliary function with the second index parameter $\rho$. Before stating the theorem and its corollary, we need the following notation: let

$$
\begin{aligned}
& P_{n}^{0}=\frac{1}{k_{0}} \sum_{i=0}^{k_{0}-1} \log Y_{i}-1, \\
& Q_{n}^{0}=\frac{1}{k_{0}} \sum_{i=0}^{k_{0}-1} \log ^{2} Y_{i}-2, \\
& P_{n}=\frac{1}{k_{0}} \sum_{i=0}^{k_{0}-1}\left(1-Y_{i}^{\gamma}\right)+\gamma /(1-\gamma), \quad \gamma<0, \\
& Q_{n}=\frac{1}{k_{0}} \sum_{i=0}^{k_{0}-1}\left(1-Y_{i}^{\gamma}\right)^{2}-2 \gamma^{2} /(1-\gamma)(1-2 \gamma), \quad \gamma<0, \\
& s^{2}(\gamma)= \begin{cases}1+\gamma^{2}, & \gamma \geq 0, \\
(1-\gamma)^{2}(1-2 \gamma)\left\{4-8 \frac{1-2 \gamma}{1-3 \gamma}+\frac{(5-11 \gamma)(1-2 \gamma)}{(1-3 \gamma)(1-4 \gamma)}\right\}, & \gamma<0,\end{cases} \\
& C\left(k_{0}, k, \rho\right)= \begin{cases}\frac{\gamma-\gamma \rho+\rho}{\gamma(1-\rho)^{2}} b\left(\frac{n}{k}\right)\left(\frac{k_{0}}{k}\right)^{-\rho}+\left(\frac{\gamma}{1+\gamma}\right)^{2}\left(\frac{k_{0}}{k}\right)^{\gamma}, & \gamma>0, \\
\frac{\rho}{(1-\rho)^{2}} b\left(\frac{n}{k_{0}}\right)+\frac{1}{\log \frac{k}{k_{0}}}, & \gamma=0, \\
\frac{(1-\gamma)(1-2 \gamma)(\gamma+\rho)}{\gamma(1-\gamma-\rho)(1-2 \gamma-\rho)} b\left(\frac{n}{k_{0}}\right)+\frac{-\gamma}{1-\gamma}\left(\frac{k_{0}}{k}\right)^{-\gamma}, & \gamma<0,\end{cases} \\
& e(\gamma)= \begin{cases}-3, & \gamma \geq 0, \\
-\left[(\gamma-1)+\frac{8(1-2 \gamma)}{1-3 \gamma}-\frac{2(1-\gamma)^{2}(5-11 \gamma)}{(1-3 \gamma)(1-4 \gamma)}\right], & \gamma<0,\end{cases}
\end{aligned}
$$




$$
\begin{aligned}
& W_{n}= \begin{cases}\frac{1}{\sigma(\gamma)} \sqrt{k_{0}}\left[(\gamma-2) P_{n}^{0}+\frac{1}{2} Q_{n}^{0}\right], & \gamma \geq 0, \\
\frac{(1-\gamma)^{2}(1-2 \gamma)}{\gamma \sigma(\gamma)} \sqrt{k_{0}}\left[2 P_{n}+\frac{1-2 \gamma}{2 \gamma} Q_{n}\right], & \gamma<0,\end{cases} \\
& d(\gamma)= \begin{cases}\alpha_{1}(\gamma), & \gamma \geq 0 \\
\alpha_{2}(\gamma), & \gamma<0\end{cases} \\
& \alpha_{1}(\gamma)=\frac{1}{\sigma^{3}(\gamma)}\left\{\left[(\gamma-2)^{3}+6(\gamma-2)^{2}\right]+12(\gamma-2)+15\right. \\
& \left.-(\gamma-1)\left[(\gamma-2)^{2}+3(\gamma-2)+3\right]+\frac{1}{3}(\gamma-1)^{3}\right\}, \\
& \alpha_{2}(\gamma)=\frac{(1-\gamma)^{2}(1-2 \gamma)^{3}}{\gamma \sigma(\gamma)} \\
& \times\left\{\frac{4}{3}\left(1+\frac{3(1-2 \gamma)}{4(1-\gamma)}\right)\left(1-\frac{3}{1-\gamma}+\frac{3}{1-2 \gamma}-\frac{1}{1-3 \gamma}\right)\right. \\
& +\frac{(1-2 \gamma)}{\gamma}\left(1-\frac{1-2 \gamma}{8(1-\gamma)}\right)\left(1-\frac{4}{1-\gamma}+\frac{6}{1-2 \gamma}-\frac{4}{1-3 \gamma}+\frac{1}{1-4 \gamma}\right) \\
& +\left(\frac{1-2 \gamma}{2 \gamma}\right)^{2}\left(1-\frac{5}{1-\gamma} \frac{10}{1-2 \gamma}-\frac{10}{1-3 \gamma}+\frac{5}{1-4 \gamma}-\frac{1}{1-5 \gamma}\right) \\
& +\frac{1}{6}\left(\frac{1-2 \gamma}{2 \gamma}\right)^{3} \\
& \times\left(1-\frac{6}{1-\gamma}+\frac{15}{1-2 \gamma}-\frac{20}{1-3 \gamma}+\frac{15}{1-4 \gamma}-\frac{6}{1-5 \gamma}+\frac{1}{1-6 \gamma}\right) \\
& \left.+\frac{4 \gamma^{3}}{(1-\gamma)^{2}(1-2 \gamma)}-\frac{1}{3}\left(\frac{\gamma}{1-\gamma}\right)^{3}\right\} \\
& A_{i}(\gamma)= \begin{cases}(\gamma-2) \log Y_{i}+\frac{1}{2} \log ^{2} Y_{i}, & \gamma \geq 0 \\
2\left(1-Y_{i}^{\gamma}\right)+\frac{1-2 \gamma}{2 \gamma}\left(1-Y_{i}^{\gamma}\right)^{2}, & \gamma<0 .\end{cases}
\end{aligned}
$$

We also need the following lemmas.

Lemma 2.1. Let $P_{n}^{0}, Q_{n}^{0}, P_{n}$, and $Q_{n}$ be as defined above.

(i) For $\gamma<0$,

$$
\sqrt{k_{0}}\left(P_{n}^{0}, Q_{n}^{0}\right) \stackrel{d}{\rightarrow} N\left(\left(\begin{array}{l}
0 \\
0
\end{array}\right),\left(\begin{array}{cc}
1 & 4 \\
4 & 20
\end{array}\right)\right)
$$

as $k_{0} \rightarrow \infty$.

(ii) For $\gamma<0$,

$$
\sqrt{k_{0}}\left(P_{n}, Q_{n}\right) \stackrel{d}{\rightarrow} N\left(\left(\begin{array}{l}
0 \\
0
\end{array}\right), \Sigma\right)
$$

as $k_{0} \rightarrow \infty$, where

$$
\Sigma=\frac{\gamma^{2}}{(1-\gamma)^{2}(1-2 \gamma)}\left(\begin{array}{cc}
1 & -\frac{4 \gamma}{1-3 \gamma} \\
-\frac{4 \gamma}{1-3 \gamma} & \frac{4 \gamma^{2}(5-11 \gamma)}{(1-2 \gamma)(1-3 \gamma)(1-4 \gamma)}
\end{array}\right) .
$$

Proof. See Dekkers et al. [4, Lemma 3.4]. 
Lemma 2.2. For every integer $m$,

$$
\mathrm{P}\left(W_{n} \leq x\right)=\Phi(x)+\sum_{v=1}^{m} Q_{v}(x) k^{-v / 2}+o\left(k^{-m / 2}\right)
$$

holds uniformly for $x \in \mathbb{R}$, where

$$
Q_{v}(x)=-\phi(x) \sum H_{v+2 s-1}(x) \prod_{j=1}^{v} \frac{1}{h_{j} !}\left(\frac{r_{j+2}}{(j+2) !}\right)^{h_{j}},
$$

the sum is over all nonnegative integer solutions of the equations

$$
h_{1}+2 h_{2}+\cdots+v h_{v}=v \quad \text { and } \quad s=h_{1}+\cdots+h_{v},
$$

$H_{l}$ is given by

$$
H_{l}(x)=l ! \sum_{i=0}^{[l / 2]} \frac{(-1)^{i} x^{l-2 i}}{i !(l-2 i) ! 2^{i}}
$$

and $r_{j}$ is the $j$ th semi-invariable of $\left\{\operatorname{Var}\left(A_{1}(\gamma)\right)\right\}^{-1 / 2}\left\{A_{1}(\gamma)-\mathrm{E} A_{1}(\gamma)\right\}$.

Proof. This follows from Theorem 4 in Petrov [15].

Theorem 2.1 concerns the expansion for the proposed estimator under general conditions.

Theorem 2.1. Suppose (1.1) holds and $U(t)$ satisfies one of conditions (2.1), (2.2), or (2.3).

(i) For $\gamma>0$,

$$
\begin{aligned}
\hat{\gamma}_{n}^{M}\left(k_{0}, k\right)= & \gamma+\frac{Q_{n}^{0}}{2}+(\gamma-2) P_{n}^{0}+\frac{\gamma-\gamma \rho+\rho}{\gamma(1-\rho)^{2}} b\left(\frac{n}{k}\right)\left(\frac{k_{0}}{k}\right)^{-\rho} \\
& +\left(\frac{\gamma}{1+\gamma}\right)^{2}\left(\frac{k_{0}}{k}\right)^{\gamma}-\frac{3}{k_{0}}+R_{1} .
\end{aligned}
$$

(ii) For $\gamma=0$,

$$
\hat{\gamma}_{n}^{M}\left(k_{0}, k\right)=\frac{Q_{n}^{0}}{2}-2 P_{n}^{0}+\frac{\rho}{(1-\rho)^{2}} b\left(\frac{n}{k_{0}}\right)+\frac{1}{\log Y_{k-k_{0}, k}}-\frac{3}{k_{0}}+R_{0} .
$$

(iii) For $\gamma<0$,

$$
\begin{aligned}
\hat{\gamma}_{n}^{M}\left(k_{0}, k\right)= & \gamma+\frac{(1-\gamma)^{2}(1-2 \gamma)}{\gamma}\left(2 P_{n}+\frac{1-2 \gamma}{2 \gamma} Q_{n}\right) \\
& +\frac{(1-\gamma)(1-2 \gamma)(\gamma+\rho)}{\gamma(1-\gamma-\rho)(1-2 \gamma-\rho)} b\left(\frac{n}{k_{0}}\right)+\frac{-\gamma}{1-\gamma}\left(\frac{k_{0}}{k}\right)^{-\gamma} \\
& +\left[(\gamma-1)+\frac{8(1-2 \gamma)}{1-3 \gamma}-\frac{2(1-\gamma)^{2}(5-11 \gamma)}{(1-3 \gamma)(1-4 \gamma)}\right] \frac{1}{k_{0}}+R_{-1} .
\end{aligned}
$$

Here, the $R_{i}, i=1,0,-1$, can be unified into an $R_{n}^{*}$ satisfying

$$
\left|R_{n}^{*}\right| \leq k_{0}^{-1}\left|H\left(\frac{1}{k_{0}}, b\left(\frac{n}{k_{0}}\right),\left(\frac{k_{0}}{k}\right)^{|\gamma|}, \frac{1}{\log Y_{k-k_{0}, k}}\right)\right|,
$$

where $H(w, x, y, z)$ is a polynomial with respect to $w, x, y$, and $z$. 
Theorem 2.2. Let $k=k(n) \rightarrow \infty, k_{0}=k_{0}(n) \rightarrow \infty, k / n \rightarrow 0, k_{0} / k \rightarrow 0$, and $U(t)$ satisfies one of conditions (2.1), (2.2), or (2.3). If there exist a sequence $\left\{B_{n}\right\}$ and a nondegenerate d.f. $\widetilde{G}$ such that

$$
\mathrm{P}\left\{B_{n}\left(\hat{\gamma}_{n}^{M}\left(k_{0}, k\right)-\gamma\right) \leq x\right\} \stackrel{d}{\rightarrow} \widetilde{G}(x),
$$

then $\widetilde{G}$ must be a normal distribution function.

Theorem 2.3. Under the assumptions of Theorem 2.2, one has

$$
\widetilde{G}=N\left(\lambda, \sigma^{2}\right) \Leftrightarrow\left\{\begin{array}{l}
\frac{B_{n}}{\sqrt{k_{0}}} \rightarrow \frac{\sigma}{\sigma(\gamma)}, \\
\sqrt{k_{0}} C\left(k_{0}, k, \rho\right) \rightarrow \frac{\lambda \sigma(\gamma)}{\sigma} .
\end{array}\right.
$$

Theorem 2.4. Under the assumptions of Theorem 2.2, one has

$$
\begin{aligned}
& \mathrm{P}\left\{\frac{\sqrt{k_{0}}\left(\hat{\gamma}_{n}^{M}\left(k_{0}, k\right)-\gamma\right)}{\sigma(\gamma)} \leq x\right\} \\
& \quad=\Phi(x)+k_{0}^{-1 / 2}\left\{d(\gamma)\left(1-x^{2}\right)-\frac{1}{\sigma(\gamma)}[\zeta+e(\gamma)]\right\} \phi(x)+o\left(k_{0}^{-1 / 2}\right)
\end{aligned}
$$

holding uniformly for $x \in \mathbb{R}$ with $k_{0}$ given by $k_{0} C\left(k_{0}, k, \rho\right) \rightarrow \zeta$.

Theorem 2.5. If we replace $k_{0} C\left(k_{0}, k, \rho\right) \rightarrow \zeta$ in Theorem 2.4 by $\sqrt{k_{0}} C\left(k_{0}, k, \rho\right) \rightarrow \zeta_{1}$, then

$$
\mathrm{P}\left\{\sqrt{k_{0}}\left(\hat{\gamma}_{n}^{M}\left(k_{0}, k\right)-\gamma\right) \leq x\right\} \rightarrow N\left(\zeta_{1}, \sigma^{2}(\gamma)\right) .
$$

Proof of Theorem 2.1. We consider the three cases successively.

(i) For $\gamma>0$, (2.1) is equivalent to

$$
\frac{U(t x)}{U(t)}=x^{\gamma}+x^{\gamma} \frac{x^{\rho}-1}{\rho} b(t)(1+o(1))
$$

as $t \rightarrow \infty$, which implies

$$
\begin{aligned}
& \frac{U\left(Y_{n-i, n}\right)-U\left(Y_{n-k, n}\right)}{U\left(Y_{n-k_{0}, n}\right)-U\left(Y_{n-k, n}\right)} \\
& =\frac{U\left(Y_{n-i, n}\right) / U\left(Y_{n-k, n}\right)-1}{U\left(Y_{n-k_{0}, n}\right) / U\left(Y_{n-k, n}\right)-1} \\
& =\frac{\left(\frac{Y_{n-i, n}}{Y_{n-k, n}}\right)^{\gamma}\left[1-\left(\frac{Y_{n-i, n}}{Y_{n-k, n}}\right)^{-\gamma}+\frac{\left(\frac{Y_{n-i, n}}{Y_{n-k, n}}\right)^{\rho}-1}{\rho} b\left(Y_{n-k, n}\right)\left(1+o_{p}(1)\right)\right]}{\left(\frac{Y_{n-k_{0}, n}}{Y_{n-k, n}}\right)^{\gamma}\left[1-\left(\frac{Y_{n-k_{0}, n}}{Y_{n-k, n}}\right)^{-\gamma}+\frac{\left(\frac{Y_{n-k_{0}, n}}{Y_{n-k, n}}\right)^{\rho}-1}{\rho} b\left(Y_{n-k, n}\right)\left(1+o_{p}(1)\right)\right]} \\
& =Y_{k_{0}-i, k_{0}}^{\gamma} \frac{1-Y_{k_{0}-i, k_{0}}^{-\gamma} Y_{k-k_{0}, k}^{-\gamma}+\frac{Y_{k_{0}-i, k_{0}}^{\rho} Y_{k-k_{0}, k}^{\rho}-1}{\rho} b\left(Y_{n-k, n}\right)\left(1+o_{p}(1)\right)}{1-Y_{k-k_{0}, k}^{-\gamma}+\frac{Y_{k-k_{0}, k}^{\rho}-1}{\rho} b\left(Y_{n-k, n}\right)\left(1+o_{p}(1)\right)} \\
& =Y_{k_{0}-i, k_{0}}^{\gamma}\left[\begin{array}{c}
1+\left(1-Y_{k_{0}-i, k_{0}}^{-\gamma}\right) Y_{k-k_{0}, k}^{-\gamma}\left(1+o_{p}(1)\right) \\
\left.\quad+\frac{Y_{k_{0}-i, k_{0}}^{\rho}-1}{\rho} Y_{k-k_{0}, k}^{\rho} b\left(Y_{n-k, n}\right)\left(1+o_{p}(1)\right)\right] .
\end{array}\right.
\end{aligned}
$$


Thus,

$$
\begin{aligned}
\hat{\gamma}_{n, j}\left(k_{0}, k\right)=\frac{1}{k_{0}} \sum_{i=0}^{k_{0}-1}\left\{\log Y_{k_{0}-i, k_{0}}^{\gamma}[1\right. & +\left(1-Y_{k_{0}-i, k_{0}}^{-\gamma}\right) Y_{k-k_{0}, k}^{-\gamma} \\
& \left.\left.+\frac{Y_{k_{0}-i, k_{0}}^{\rho}-1}{\rho} Y_{k-k_{0}, k}^{\rho} b\left(Y_{n-k, n}\right)+r_{n, i}\right]\right\}^{j} \\
=\frac{1}{k_{0}} \sum_{i=0}^{k_{0}-1}\left\{\gamma \log Y_{k_{0}-i, k_{0}}+\right. & \left(1-Y_{k_{0}-i, k_{0}}^{-\gamma}\right) Y_{k-k_{0}, k}^{-\gamma} \\
& \left.+\frac{Y_{k_{0}-i, k_{0}}^{\rho}-1}{\rho} Y_{k-k_{0}, k}^{\rho} b\left(Y_{n-k, n}\right)+r_{n, i}\right\}^{j} .
\end{aligned}
$$

In particular,

$$
\begin{aligned}
& \hat{\gamma}_{n, 1}\left(k_{0}, k\right)=\gamma+\gamma P_{n}^{0}+\frac{\gamma}{\gamma+1}\left(\frac{k_{0}}{k}\right)^{\gamma}+\frac{1}{1-\rho}\left(\frac{k_{0}}{k}\right)^{-\rho} b\left(\frac{n}{k}\right)+R_{11}, \\
& \hat{\gamma}_{n, 2}\left(k_{0}, k\right)=\frac{1}{k_{0}} \sum_{i=0}^{k_{0}-1}\left\{\gamma^{2} \log ^{2} Y_{k_{0}-i, k_{0}}+2 \gamma\left(1-Y_{k_{0}-i, k_{0}}^{-\gamma}\right) \log Y_{k_{0}-i, k_{0}} Y_{k-k_{0}, k}^{-\gamma}\right. \\
&\left.+2 \gamma \frac{Y_{k_{0}-i, k_{0}}^{\rho}-1}{\rho} \log Y_{k_{0}-i, k_{0}} Y_{k-k_{0}, k}^{\rho} b\left(Y_{n-k, n}\right)+r_{n, i}^{*}\right\} \\
&=2 \gamma^{2}+\gamma^{2} Q_{n}^{0}+\frac{2 \gamma}{\rho}\left(\frac{1}{(1-\rho)^{2}}-1\right)\left(\frac{k_{0}}{k}\right)^{-\rho} b\left(\frac{n}{k}\right) \\
&-2 \gamma\left(\frac{1}{(1+\gamma)^{2}}-1\right)\left(\frac{k_{0}}{k}\right)^{\gamma}+R_{12}, \\
& \hat{\gamma}_{n, 1}^{2}\left(k_{0}, k\right)= \gamma^{2}+2 \gamma^{2} P_{n}^{0}+\frac{2 \gamma}{1-\rho}\left(\frac{k_{0}}{k}\right)^{-\rho} b\left(\frac{n}{k}\right)+\frac{2 \gamma^{2}}{1+\gamma}\left(\frac{k_{0}}{k}\right)^{\gamma}+\gamma^{2} P_{n}^{02}+R_{11},
\end{aligned}
$$

where both $R_{11}$ and $R_{12}$ are $o\left(\left(k_{0} / k\right)^{\gamma}, Y_{k-k_{0}, k}^{\rho} b\left(Y_{n-k, n}\right)\right)$. Hence,

$$
\begin{aligned}
\hat{\gamma}_{n, 2}-\hat{\gamma}_{n, 1}^{2}= & \gamma^{2}+\gamma^{2}\left(Q_{n}^{0}-2 P_{n}^{0}\right)+\frac{2 \gamma}{\rho}\left(\frac{1}{(1-\rho)^{2}}-\frac{1}{1-\rho}\right)\left(\frac{k_{0}}{k}\right)^{-\rho} b\left(\frac{n}{k}\right) \\
-2 \gamma & \left(\frac{1}{(1+\gamma)^{2}}-\frac{1}{1+\gamma}\right)\left(\frac{k_{0}}{k}\right)^{\gamma}-\gamma^{2} P_{n}^{02}+R^{11} \\
\hat{\gamma}_{n, 2}-2 \hat{\gamma}_{n, 1}^{2}= & \gamma^{2}\left(Q_{n}^{0}-4 P_{n}^{0}\right)+\frac{2 \gamma}{\rho}\left(\frac{1}{1-\rho}-1\right)^{2}\left(\frac{k_{0}}{k}\right)^{-\rho} b\left(\frac{n}{k}\right) \\
& -2 \gamma\left(\frac{1}{1+\gamma}-1\right)^{2}\left(\frac{k_{0}}{k}\right)^{\gamma}-2 \gamma^{2} P_{n}^{02}+R^{12} .
\end{aligned}
$$

The result for $\hat{\gamma}_{n}^{M}\left(k_{0}, k\right)$ follows by combining these two relations.

(ii) For $\gamma=0$, note that

$$
\begin{aligned}
\frac{1}{b(t)} & \left\{\frac{U(t x)-U(t)}{a(t)}-\log x\right\} \rightarrow \frac{x^{\rho}-1}{\rho} \\
& \Longleftrightarrow \frac{U(t x)-U(t y)}{U(t y)-U(t)}=\frac{\log \frac{x}{y}+\frac{\left(\frac{x}{y}\right)^{\rho}-1}{\rho} b(t y)(1+o(1))}{\log y-\frac{y^{-\rho}-1}{\rho} b(t y)(1+o(1))}
\end{aligned}
$$


and so

$$
\begin{aligned}
\frac{U\left(Y_{n-i, n}\right)-U\left(Y_{n-k, n}\right)}{U\left(Y_{n-k_{0}, n}\right)-U\left(Y_{n-k, n}\right)} & =1+\frac{U\left(Y_{n-i, n}\right)-U\left(Y_{n-k_{0}, n}\right)}{U\left(Y_{n-k_{0}, n}\right)-U\left(Y_{n-k, n}\right)} \\
& =1+\frac{U\left(Y_{k_{0}-i, k_{0}} Y_{n-k, n}\right)-U\left(Y_{k-k_{0}, k} Y_{n-k, n}\right)}{U\left(Y_{k-k_{0}, k} Y_{n-k, n}\right)-U\left(Y_{n-k, n}\right)} \\
& =1+\frac{\log Y_{k_{0}-i, k_{0}}+\frac{Y_{k_{0}-i, k_{0}}^{\rho}-1}{\rho} b\left(Y_{n-k_{0}, n}\right)\left(1+o_{p}(1)\right)}{\log Y_{k-k_{0}, k}-\frac{Y_{k_{0}-i, k_{0}}-1}{\rho} b\left(Y_{n-k_{0}, n}\right)\left(1+o_{p}(1)\right)} \\
& =1+\frac{u_{1}\left(k_{0}, k\right)}{u_{2}\left(k_{0}, k\right)} .
\end{aligned}
$$

Thus,

$$
\begin{aligned}
\hat{\gamma}_{n, j}\left(k_{0}, k\right) & =\frac{1}{k_{0}} \sum_{i=0}^{k_{0}-1}\left\{\log \left[1+\frac{u_{1}\left(k_{0}, k\right)}{u_{2}\left(k_{0}, k\right)}\right]\right\} \\
& =\frac{1}{k_{0}} \sum_{i=0}^{k_{0}-1}\left\{\log ^{-1} Y_{k-k_{0}, k} u_{1}\left(k_{0}, k\right)\right\}^{j} \\
& =\log ^{-j} Y_{k-k_{0}, k} \frac{1}{k_{0}} \sum_{i=0}^{k_{0}-1} u_{1}^{j}\left(k_{0}, k\right) .
\end{aligned}
$$

In particular,

$$
\begin{gathered}
\hat{\gamma}_{n, 1}\left(k_{0}, k\right)=\log ^{-1} Y_{k-k_{0}, k}\left[1+P_{n}^{0}+\frac{1}{1-\rho} b\left(\frac{n}{k_{0}}\right)+R_{01}\right], \\
\hat{\gamma}_{n, 2}\left(k_{0}, k\right)=\log ^{-2} Y_{k-k_{0}, k}\left[2+Q_{n}^{0}+\frac{2(2-\rho)}{(1-\rho)^{2}} b\left(\frac{n}{k_{0}}\right)+R_{02}\right], \\
\hat{\gamma}_{n, 1}^{2}\left(k_{0}, k\right)=\log ^{-2} Y_{k-k_{0}, k}\left[1+2 P_{n}^{0}+\frac{2}{1-\rho} b\left(\frac{n}{k_{0}}\right)+P_{n}^{02}+R_{01}\right] .
\end{gathered}
$$

Hence,

$$
\begin{aligned}
\hat{\gamma}_{n, 2}- & \hat{\gamma}_{n, 1}^{2} \\
= & \log ^{-2} Y_{k-k_{0}, k}\left[1+\left(Q_{n}^{0}-2 P_{n}^{0}\right)+2\left(\frac{2-\rho}{(1-\rho)^{2}}-\frac{1}{1-\rho}\right) b\left(\frac{n}{k_{0}}\right)-P_{n}^{02}+R^{01}\right], \\
\hat{\gamma}_{n, 2}- & 2 \hat{\gamma}_{n, 1}^{2} \\
& =\log ^{-2} Y_{k-k_{0}, k}\left[\left(Q_{n}^{0}-4 P_{n}^{0}\right)+2\left(\frac{2-\rho}{(1-\rho)^{2}}-\frac{2}{1-\rho}\right) b\left(\frac{n}{k_{0}}\right)-2 P_{n}^{02}+R^{02}\right] .
\end{aligned}
$$

The result for $\hat{\gamma}_{n}^{M}\left(k_{0}, k\right)$ follows by combining these two relations.

(iii) For $\gamma<0$, note that $V(t)=U(\infty)-U(t) \in \mathbb{R}$ and

$$
\begin{aligned}
& \left\{\frac{V(t x)}{V(t)}-x^{\gamma}\right\} \frac{1}{b(t)} \rightarrow x^{\gamma} \frac{x^{\rho}-1}{\rho} \\
& \Longleftrightarrow \frac{V(t x)}{V(t)}=x^{\gamma}+x^{\gamma} \frac{x^{\rho}-1}{\rho} b(t)(1+o(1))
\end{aligned}
$$


and so

$$
\begin{aligned}
& \frac{U\left(Y_{n-i, n}\right)-U\left(Y_{n-k, n}\right)}{U\left(Y_{n-k_{0}, n}\right)-U\left(Y_{n-k, n}\right)} \\
& =1+\frac{U\left(Y_{n-i, n}\right)-U\left(Y_{n-k_{0}, n}\right)}{U\left(Y_{n-k_{0}, n}\right)-U\left(Y_{n-k, n}\right)} \\
& =1+\frac{V\left(Y_{k_{0}-i, k_{0}} Y_{n-k, n}\right)-V\left(Y_{k-k_{0}, k} Y_{n-k, n}\right)}{V\left(Y_{k-k_{0}, k} Y_{n-k, n}\right)-V\left(Y_{n-k, n}\right)} \\
& =1+\frac{\frac{V\left(Y_{k_{0}-i, k_{0}} Y_{n-k, n}\right)}{V\left(Y_{k-k_{0}, k} Y_{n-k, n}\right)}-1}{1-\frac{V\left(Y_{k-k_{0}, k} Y_{n-k, n}\right)}{V\left(Y_{n-k, n}\right)}} \\
& =1+\frac{\left(\frac{Y_{n-i, n}}{Y_{n-k_{0}, n}}\right)^{\gamma}+\left(\frac{Y_{n-i, n}}{Y_{n-k_{0}, n}}\right)^{\gamma} \frac{\left(\frac{Y_{n-i, n}}{Y_{n-k_{0}, n}}\right)^{\rho}-1}{\rho} b\left(Y_{n-k_{0}, n}\right)\left(1+o_{p}(1)\right)-1}{1-\left(\frac{Y_{n-k, n}}{Y_{n-k_{0}, n}}\right)^{\gamma}-\left(\frac{Y_{n-k, n}}{Y_{n-k_{0}, n}}\right)^{\gamma} \frac{\left(\frac{Y_{n-k, n}}{Y_{n-k_{0}, n}}\right)^{\rho}-1}{\rho} b\left(Y_{n-k_{0}, n}\right)\left(1+o_{p}(1)\right)} \\
& =1+\frac{Y_{k_{0}-i, k_{0}}^{\gamma}+Y_{k_{0}-i, k_{0}}^{\gamma} \frac{Y_{k_{0}-i, k_{0}}^{\rho}-1}{\rho} b\left(Y_{n-k_{0}, n}\right)\left(1+o_{p}(1)\right)-1}{1-Y_{k-k_{0}, k}^{-\gamma}-Y_{k-k_{0}, k}^{-\gamma} \frac{Y_{k-k_{0}, k}^{-1}}{\rho} b\left(Y_{n-k_{0}, n}\right)\left(1+o_{p}(1)\right)} \\
& =1+Y_{k-k_{0}, k}^{\gamma}\left[1-Y_{k_{0}-i, k_{0}}^{\gamma}-Y_{k_{0}-i, k_{0}}^{\gamma} \frac{Y_{k_{0}-i, k_{0}}^{\rho}-1}{\rho} b\left(Y_{n-k_{0}, n}\right)\left(1+o_{p}(1)\right)\right] .
\end{aligned}
$$

Thus,

$$
\begin{aligned}
& \hat{\gamma}_{n, j}\left(k_{0}, k\right)=\frac{1}{k_{0}} \sum_{i=0}^{k_{0}-1}\left\{\operatorname { l o g } \left[1+Y_{k-k_{0}, k}^{\gamma}\left(1-Y_{k_{0}-i, k_{0}}^{\gamma}\right.\right.\right. \\
&- Y_{k_{0}-i, k_{0}}^{\gamma} \frac{Y_{k_{0}-i, k_{0}}^{\rho}-1}{\rho} \\
&\left.\left.\left.\times b\left(Y_{n-k_{0}, n}\right)\left(1+o_{p}(1)\right)\right)\right]\right\}^{j} \\
&=\frac{1}{k_{0}} \sum_{i=0}^{k_{0}-1}\left\{Y _ { k - k _ { 0 } , k } ^ { \gamma } \left(1-Y_{k_{0}-i, k_{0}}^{\gamma}\right.\right. \\
&\left.\left.-Y_{k_{0}-i, k_{0}}^{\gamma} \frac{Y_{k_{0}-i, k_{0}}^{\rho}-1}{\rho} b\left(Y_{n-k_{0}, n}\right)\left(1+o_{p}(1)\right)\right)\right\}^{j} .
\end{aligned}
$$

In particular,

$$
\begin{gathered}
\hat{\gamma}_{n, 1}\left(k_{0}, k\right)=Y_{k-k_{0}, k}^{\gamma}\left[\frac{-\gamma}{1-\gamma}+P_{n}-\frac{1}{(1-\gamma)(1-\gamma-\rho)} b\left(\frac{n}{k_{0}}\right)+R_{-11}\right], \\
\hat{\gamma}_{n, 2}\left(k_{0}, k\right)=Y_{k-k_{0}, k}^{2 \gamma}\left[\frac{2 \gamma^{2}}{(1-\gamma)(1-2 \gamma)}+Q_{n}+R_{-12}\right. \\
\left.\quad-2\left[\frac{1}{(1-\gamma)(1-\gamma-\rho)}-\frac{1}{(1-2 \gamma)(1-2 \gamma-\rho)}\right] b\left(\frac{n}{k_{0}}\right)\right],
\end{gathered}
$$


and

$$
\begin{aligned}
\hat{\gamma}_{n, 1}^{2}\left(k_{0}, k\right)=Y_{k-k_{0}, k}^{2 \gamma}[ & \left(\frac{\gamma}{1-\gamma}\right)^{2}-\frac{2 \gamma}{1-\gamma} P_{n} \\
& \left.+\frac{2 \gamma}{(1-\gamma)^{2}(1-\gamma-\rho)} b\left(\frac{n}{k_{0}}\right)+P_{n}^{2}+R_{-11}\right] .
\end{aligned}
$$

Hence,

$$
\begin{aligned}
& \hat{\gamma}_{n, 2}-\hat{\gamma}_{n, 1}^{2}=Y_{k-k_{0}, k}^{2 \gamma}\{ \frac{2 \gamma^{2}}{(1-\gamma)^{2}(1-2 \gamma)}+\left(Q_{n}+\frac{2 \gamma}{1-\gamma} P_{n}\right) b\left(\frac{n}{k_{0}}\right)-P_{n}^{2}+R^{-11} \\
&-2\left[\frac{1}{(1-\gamma)(1-\gamma-\rho)}-\frac{1}{(1-2 \gamma)(1-2 \gamma-\rho)}\right. \\
& \hat{\gamma}_{n, 2}-2 \hat{\gamma}_{n, 1}^{2}=Y_{k-k_{0}, k}^{2 \gamma}\left\{\frac{2 \gamma^{2} \gamma}{(1-\gamma)^{2}(1-2 \gamma)}+\left(Q_{n}+\frac{4 \gamma}{1-\gamma} P_{n}\right)-2 P_{n}^{2}+R^{-12}\right. \\
&\left.-2\left[\frac{1}{(1-\gamma)(1-\gamma-\rho)}-\frac{1}{(1-2 \gamma)(1-2 \gamma-\rho)}\right]\right\}, \\
&\left.\left.+\frac{2 \gamma}{(1-\gamma)^{2}(1-\gamma-\rho)}\right] b\left(\frac{n}{k_{0}}\right)\right\} .
\end{aligned}
$$

The result for $\hat{\gamma}_{n}^{M}\left(k_{0}, k\right)$ follows by combining these two relations.

Proof of Theorem 2.2. Since

$$
\begin{aligned}
\mathrm{P}\left\{B_{n}\left(\hat{\gamma}_{n}^{M}\left(k_{0}, k\right)-\gamma\right) \leq x\right\} \\
\quad=\mathrm{P}\left\{B_{n}\left(\frac{\sigma(\gamma)}{\sqrt{k_{0}}} W_{n}+C\left(k_{0}, k, \rho\right)+e(\gamma) \frac{1}{k_{0}}+R_{n}^{*}\right) \leq x\right\} \\
\quad=\mathrm{P}\left\{\frac{\sigma(\gamma)}{\sqrt{k_{0}}} W_{n}+C\left(k_{0}, k, \rho\right)+e(\gamma) \frac{1}{k_{0}}+R_{n}^{*} \leq \frac{x}{B_{n}}\right\},
\end{aligned}
$$

by Lemma 2.2 , one can bound

$$
\begin{aligned}
\sup _{x} \mid \mathrm{P}\left\{B_{n}\left(\hat{\gamma}_{n}^{M}\left(k_{0}, k\right)-\gamma\right) \leq x\right\} \\
\quad-\mathrm{P}\left\{B_{n}\left(\frac{\sigma(\gamma)}{\sqrt{k_{0}}} N+C\left(k_{0}, k, \rho\right)+e(\gamma) \frac{1}{k_{0}}+R_{n}^{*}\right) \leq x\right\} \mid \leq O\left(k_{0}^{-1 / 2}\right) .
\end{aligned}
$$

This means that $B_{n}\left(\hat{\gamma}_{n}^{M}\left(k_{0}, k\right)-\gamma\right)$ has a nondegenerate limiting d.f. if and only if

$$
\frac{B_{n}}{\sqrt{k_{0}}} \sigma(\gamma) N+B_{n} C\left(k_{0}, k, \rho\right)+B_{n} e(\gamma) \frac{1}{k_{0}}+B_{n} R_{n}^{*}
$$

has the same nondegenerate limiting d.f. Under the assumptions of Theorem 2.2, the 
limiting d.f. of (2.5) is $\widetilde{G}$, and

$$
\begin{gathered}
\limsup _{n \rightarrow \infty} \frac{B_{n}}{\sqrt{k_{0}}} \sigma(\gamma)<\infty, \\
\limsup _{n \rightarrow \infty}\left(B_{n} C\left(k_{0}, k, \rho\right)+B_{n} e(\gamma) \frac{1}{k_{0}}+B_{n} R_{n}^{*}\right)<\infty .
\end{gathered}
$$

Thus, there exist a subsequence $\left\{n^{\prime}\right\}$ and constants $(\lambda, \sigma)$ such that

$$
\begin{gathered}
\lim _{n \rightarrow \infty} \frac{B_{n^{\prime}}}{\sqrt{k_{0}\left(n^{\prime}\right)}} \sigma(\gamma)=\sigma \\
\lim _{n \rightarrow \infty} B_{n^{\prime}} C\left(k_{0}\left(n^{\prime}\right), k\left(n^{\prime}\right), \rho\right)+B_{n^{\prime}} e(\gamma) \frac{1}{k_{0}\left(n^{\prime}\right)}+B_{n^{\prime}} R_{n^{\prime}}^{*}=\lambda
\end{gathered}
$$

with $\widetilde{G}$ nondegenerate, which implies $\sigma \neq 0$. Hence, $\widetilde{G}$ is the normal d.f. $N\left(\lambda, \sigma^{2}\right)$.

Proof of Theorem 2.3. $(\Longleftarrow)$ If $(2.4)$ holds, then

$$
\lim _{n \rightarrow \infty} \frac{B_{n}}{\sqrt{k_{0}}} \sigma(\gamma)=\sigma
$$

and

$$
\lim _{n \rightarrow \infty} B_{n} C\left(k_{0}, k, \rho\right)+B_{n} e(\gamma) \frac{1}{k_{0}}+B_{n} R_{n}^{*}=\lambda
$$

hold, implying that (2.5) converges to $s N+\lambda$ in distribution, i.e.,

$$
B_{n}\left(\hat{\gamma}_{n}^{M}\left(k_{0}, k\right)-\gamma\right) \stackrel{d}{\rightarrow} N\left(\lambda, \sigma^{2}\right) .
$$

$(\Longrightarrow)$ If (2.7) holds, then from the proof of Theorem 2.2, one has

$$
\lim _{n \rightarrow \infty} \frac{B_{n}}{\sqrt{k_{0}}} \sigma(\gamma)=\sigma
$$

and

$$
\lim _{n \rightarrow \infty} B_{n} C\left(k_{0}, k, \rho\right)+B_{n} e(\gamma) \frac{1}{k_{0}}+B_{n} R_{n}^{*}=\lambda .
$$

By the property of $R_{n}^{*},(2.9)$ is equivalent to

$$
\lim _{n \rightarrow \infty} B_{n} C\left(k_{0}, k, \rho\right) \rightarrow \lambda,
$$

which together with (2.8) completes the proof.

Proof of Theorem 2.4. By Theorem 2.1,

$$
\hat{\gamma}_{n}^{M}\left(k_{0}, k\right)=\gamma+\frac{\sigma(\gamma)}{\sqrt{k_{0}}} W_{n}+C\left(k_{0}, k, \rho\right)+e(\gamma) \frac{1}{k_{0}}+R_{n}^{*} .
$$

Thus, one can write

$$
\begin{aligned}
\sqrt{k_{0}}\left(\hat{\gamma}_{n}^{M}\left(k_{0}, k\right)-\gamma\right) & =\sqrt{k_{0}}\left(\frac{\sigma(\gamma)}{\sqrt{k_{0}}} W_{n}+C\left(k_{0}, k, \rho\right)+e(\gamma) \frac{1}{k_{0}}+R_{n}^{*}\right) \\
& =\sigma(\gamma) W_{n}+\left[k_{0} C\left(k_{0}, k, \rho\right)+e(\gamma)\right] k_{0}^{-1 / 2}+o\left(k_{0}^{-1 / 2}\right) \\
& =\sigma(\gamma) W_{n}+[\zeta+e(\gamma)] k_{0}^{-1 / 2}+o\left(k_{0}^{-1 / 2}\right)
\end{aligned}
$$


and now application of Lemma 2.2 shows that

$$
\begin{aligned}
& \mathrm{P}\left\{\frac{\sqrt{k_{0}}\left(\hat{\gamma}_{n}^{M}\left(k_{0}, k\right)-\gamma\right)}{\sigma(\gamma)} \leq x\right\} \\
& \quad=\mathrm{P}\left\{W_{n} \leq x-\frac{1}{\sigma(\gamma)}[\zeta+e(\gamma)] k_{0}^{-1 / 2}+o\left(k_{0}^{-1 / 2}\right)\right\} \\
& \quad=\Phi(x)-k_{0}^{-1 / 2}\left\{d(\gamma)\left(1-x^{2}\right)-\frac{1}{\sigma(\gamma)}[\zeta+e(\gamma)]\right\} \phi(x)+o\left(k_{0}^{-1 / 2}\right) .
\end{aligned}
$$

Note that

$$
\begin{gathered}
Q_{1}(x)=-\phi(x) H_{2}(x) r_{3} / 6, \\
H_{2}(x)=x^{2}-1, \\
r_{3}=\left\{\operatorname{Var}\left(A_{1}(\gamma)\right)\right\}^{-3 / 2} \mathrm{E}\left(A_{1}(\gamma)-\mathrm{E} A_{1}(\gamma)\right)^{3} .
\end{gathered}
$$

Furthermore,

$$
\begin{gathered}
\mathrm{E} A_{1}(\gamma)=\gamma-1, \\
\mathrm{E}\left(A_{1}(\gamma)\right)^{2}=2(\gamma-2)^{2}+6(\gamma-2)+6, \\
\mathrm{E}\left(A_{1}(\gamma)\right)^{3}=6(\gamma-2)^{3}+36(\gamma-2)^{2}+90(\gamma-2)+90
\end{gathered}
$$

for $\gamma \geq 0$ and

$$
\begin{gathered}
\mathrm{E} A_{1}(\gamma)=2 \mathrm{E}\left(1-R_{1}\right)+\frac{1-2 \gamma}{2 \gamma} \mathrm{E}\left(1-R_{1}\right)^{2} \\
\mathrm{E}\left(A_{1}(\gamma)\right)^{2}=4 \mathrm{E}\left(1-R_{1}\right)^{2}+\left(\frac{1-2 \gamma}{2 \gamma}\right)^{2} \mathrm{E}\left(1-R_{1}\right)^{4}+2 \frac{1-2 \gamma}{\gamma} \mathrm{E}\left(1-R_{1}\right)^{3} \\
\mathrm{E}\left(A_{1}(\gamma)\right)^{3}=8 \mathrm{E}\left(1-R_{1}\right)^{3}+6 \frac{1-2 \gamma}{\gamma} \mathrm{E}\left(1-R_{1}\right)^{4}+6\left(\frac{1-2 \gamma}{2 \gamma}\right)^{2} \mathrm{E}\left(1-R_{1}\right)^{5} \\
+\left(\frac{1-2 \gamma}{2 \gamma}\right)^{3} \mathrm{E}\left(1-R_{1}\right)^{6}
\end{gathered}
$$

for $\gamma<0$, where $R_{1}=Y_{1}^{\gamma}$. The proof of the theorem is complete upon calculating the necessary parts in Lemma 2.2 .

Proof of Theorem 2.5. The theorem easily follows from Theorems 2.1 and 2.4.

\section{BIBLIOGRAPHY}

1. S. Cheng and J. Pan, Asymptotic expansions of estimators for the tail index with applications, Scand. J. Statist. 25 (1998), 717-728. MR1666796 (2000d:62079)

2. A. Cuntz, E. Haeusler, and J. Segers, Edgeworth expansions for the distribution function of the Hill estimator, Discussion Paper, vol. 8, Center for Economic Research, Tilburg University, 2003.

3. L. de Haan and U. Statmüller, Generalized regular variation of second order, J. Austral. Math. Soc. Ser. A 61 (1996), 381-395. MR.1420345 (97g:26001)

4. A. L. M. Dekkers, J. H. J. Einmahl, and L. De Haan, A moment estimator for the index of an extreme-value distribution, Ann. Statist. 17 (1989), 1833-1855. MR.1026315 (91i:62033)

5. H. Drees, A general class of estimators of the extreme value index, J. Statist. Plann. Inference 66 (1998), 95-112. MR.1616999 (99c:62085)

6. Z. Fan, Estimation problems for distributions with heavy tails, J. Statist. Plann. Inference 123 (2004), 13-40. MR2058119 (2005c:62059)

7. M. I. Fraga Alves, A location invariant Hill-type estimator, Extremes 3 (2001), 199-217. MR $1907061(2003 \mathrm{~d}: 62129)$ 
8. M. I. Gomes and J. Martins, Generalizations of the Hill estimator-asymptotic versus finite sample behavior, J. Statist. Plann. Inference 93 (2001), 161-180. MR1822394

9. M. I. Gomes and O. Oliveira, Censoring estimators of a positive tail index, Statist. Probab. Lett. 65 (2003), 147-159. MR2018025 (2005d:62155)

10. P. Hall, On estimating the endpoint of a distribution, Ann. Statist. 3 (1982), 556-568. MR653530 (83f:62043)

11. B. M. Hill, A simple general approach to inference about the tail of a distribution, Ann. Statist. 3 (1975), 1163-1174. MR0378204 (51:14373)

12. J. Pan, Some results on estimation of the tail index of a distribution, Chin. Ann. Math. Ser. B 19 (1998), 239-248. MR.1655939 (2000a:62037)

13. L. Peng, Asymptotically unbiased estimators for the extreme value index, Statist. Probab. Lett. 38 (1998), 107-115. MR1627906 (99e:62056)

14. Z. Peng, Extension of Pickands' estimator, Acta Math. Sinica (Chin. Ser.) 40 (1997), 759-762. MR:1612631 (99c:62111)

15. V. V. Petrov, Sums of Independent Random Variables, Ergebnisse der Mathematik und ihrer Grenzgebiete, vol. 82, Springer-Verlag, New York-Heidelberg, 1975. MR0388499 (52:9335)

16. J. Pickands, Statistical inference using extreme order statistics, Ann. Statist. 3 (1975), 119-131. MR0423667 (54:11642)

17. Y. Qi and S. Cheng, Convergence of Pickands-type estimators, Chinese Sci. Bull. 37 (1992), 1409-1413.

18. J. Segers, Abelian and Tauberian theorems for the bias of the Hill estimator, Scand. J. Statist. 29 (2001), 461-483. MR1925570 (2003k:62159)

19. J. Segers, Generalized Pickands estimators for the extreme value index, J. Statist. Plann. Inference 128 (2005), 381-396. MR2102765

20. R. L. Smith, Maximum likelihood estimation in a class of nonregular cases, Biometrika 72 (1985), 67-90. MR790201 (86k:62053)

21. R. L. Smith, Estimation of tails of probability distribution, Ann. Statist. 15 (1987), 1174-1207. MR.902252 (88j:62096)

22. R. L. Smith and I. Weissman, Maximum likelihood estimation of the lower tail of probability distribution, J. Roy. Statist. Soc. Ser. B 47 (1985), 285-298. MR816094 (87b:62035)

23. Z. Tsourti and I. Panaretos, Extreme value index estimators and smoothing alternatives: review and simulation comparison, Technical Report, vol. 149, Department of Statistics, Athens University of Economics and Business, 2001.

24. X. Wang and S. Cheng, General regular variation of nth order and the second order Edgeworth expansion of the extreme value distribution. I, Acta Math. Sin. (English Ser.) 21 (2005), no. 5, 1121-1130. MR2176324 (2006i:60063)

25. S. Yun, On a general Pickands estimator of extreme value index, J. Statist. Plann. Inference 102 (2002), 389-409. MR1896495 (2003e:62050)

Department of Mathematics, Southwest Normal University, Chongqing 400715, People's REPUBlic OF China

Department of Mathematics, Southwest Normal University, Chongqing 400715, People's Republic of China

E-mail address: pzx@swu.edu.cn

Department of Statistics, University of Nebraska-Lincoln, Lincoln, Nebraska 68583

E-mail address: snadaraj@unlserve.unl.edu

Received 29/NOV/2005

Originally published in English 